DOI: $10.3901 / J M E .2015 .03 .206$

\title{
基于均匀设计法的精密车削参数优化*
}

\author{
李登万 ${ }^{1}$ 陈洪涛 $^{1}$ 冯锦春 $^{1}$ 钟成明 $^{2}$ \\ (1. 四川工程职业技术学院四川装备制造业产业集群技术创新中心＼cjkstart德阳 618099; \\ 2. 东方汽轮机有限公司叶片分厂 德阳 618099)
}

\begin{abstract}
摘要: 为了探索难加工材料-奥氏体不锈钢精密车削的参数优化问题, 引入伪变量 $\mathrm{A}$ 表达车削冷却降温条件, 用均匀设计法 设计含定性因素混合水平的精密车削试验方案。在干式、环保型湿式和低温冷风微油雾三种不同车削冷却降温条件下, 实现 低成本高效率的精密车削试验。在这三种条件下, 以切削速度、进给量、背吃刀量和刀尖半径为优化变量, 以表面粗徏度、 表面残余应力、切削力、切削温度、刀具寿命和切削效率为优化目标函数, 建立了奥氏体不锈钢精密车削参数优化模型, 还 对车削参数进行了优化和验证, 效果明显。以刀具磨钝前能车削出的总金属表面积作为刀具寿命, 在工件连续表面不出现接 刀现象的前提下进行精密车削参数优化。研究成果对指导大型工件精密车削的参数选择具有实际意义。
\end{abstract}

关键词: 均匀设计; 精密车削; 参数优化; 刀具寿命; 表面残余应力

中图分类号: TG506

\section{Precision Cutting Parameters Optimization Based on Uniform Design Method}

\author{
LI Dengwan ${ }^{1}$ CHEN Hongtao ${ }^{1}$ FENG Jinchun ${ }^{1}$ ZHONG Chengming ${ }^{2}$ \\ (1. Sichuan Technology Innovation Center of Equipment Manufacturing Industry Cluster, \\ Sichuan Engineering Technical College, Deyang 618099; \\ 2. Vane Branch, Dongfang Steam Turbine Co., Ltd., Deyang 618099)
}

\begin{abstract}
In order to explore precision turning parameter optimization of austenitic stainless steel which is a difficult cutting material, pseudo-variable $\mathrm{A}$ is introduced as a factor of cooling cutting conditions, and a precision turning experiment program is projected by uniform design method, which contains qualitative factors hybrid level. A low cost and high efficiency turning test is carried out in three different conditions of dry cutting, wet cutting and cutting with low temperature air containing micro-oil mist. Under the three cooling cutting conditions, the precision turning parameter optimization model of austenitic stainless steel is established, in which, cutting speed, feed amount, cutting depth and tool nose radius are taken as optimization variables, and surface roughness, surface residual stress, cutting force, cutting temperature, tool life and cutting efficiency are taken as objective functions. Meanwhile, the parameters are optimized and verified, and the test results show that the optimization effect is good. Total metal surface area that can be lathed before cutter blunt is taken as the cutter life, and precision turning parameters are optimized in the condition of workpiece continuous surface without machined trace. The results are useful for precision turning parameters selection in heavy workpiece cutting.
\end{abstract}

Key words: uniform design; precision turning; parameter optimization; tool life; surface residual stress

\section{0 前言}

优化问题的实质是在给定边界条件下得到相对 最优的结果。在机械制造领域, 有先进的金属切削 机床，若切削刀具、工艺装备和工艺技术等软环境 没有跟上，同样难达到理想的切削效率与切削加工

* 国家重点基础研究发展计划资助项目(973 计划, 2007CB707703-4)。 20140305 收到初稿, 20141009 收到修改稿
表面完整性。在切削加工系统中直接和工件发生干 涉关系的是刀具, 机床的高性能必须通过切削刀具 才能映射到工件的切削表面上, 为更好地发挥机床 和刀具的性能, 就应对切削相关参数进行优化。精 密车削的参数优化, 实质是控制切削力、切削温度、 表面粗糙度和表面残余应力, 确保刀具拥有合适的 寿命, 发挥机床和刀具的优良性能, 而提高切削 效率 ${ }^{[1]}$ 。

为了优化奥氏体不锈钢精密车削的相关参数, 过去人们常在切削液冷却降温条件下进行车削试验 
研究, 用正交试验法设计试验方案 ${ }^{[2]}$ 。正交试验法 的试验次数多、周期长、成本高。在现代生态文明 的大背景下, 节能降减排、提高效率, 已成为社会 普遍关心的热点问题, 不仅要研究清洁切削, 同时 试验方案也要高效。研究奥氏体不锈钢精密清洁车 削, 即在干式车削 ${ }^{[3]}$ 、低温冷风微量油雾车削 ${ }^{[4-5]}$ 和 环保型湿式车削三种冷却降温切削条件下, 用均匀 设计方法 ${ }^{[1,6-7]}$ 设计精密车削试验参数。在三种清洁 车削冷却降温条件下, 以切削速度、进给量、背吃 刀量、刀尖半径为自变量, 以表面粗䊁度、切削力、 表面残余应力、切削温度、刀具寿命和切削效率为 目标函数, 建立多元二次回归模型 ${ }^{[8-9]}$ 。在回归模型 基础之上建立满足零件表面粗䊁度和表面残余应力 要求、刀具寿命适度、切削温度与切削力较小、切 削效率最高为目标的精密车削的参数优化模 型 ${ }^{8}$, 10-12]。本模型对指导奥氏体不锈钢大型工件清洁精 密车削的参数选用具有实际工程意义。

\section{1 试验条件和方案}

试验对象：奥氏体不锈钢(022Cr19Ni10)。试验 机床: CK6143。试验刀具: CNMG120404FP、 CNMG120408FP 和 CNMG120412FP 肯纳刀片, 装 刀后的车刀几何角度: 主偏角 $\kappa_{\mathrm{r}}=93^{\circ}$ 、副偏角 $\kappa_{\mathrm{r}}^{\prime}=7^{\circ}$ 、刃倾角 $\lambda_{\mathrm{s}}=-8^{\circ}$ 、前角 $\gamma_{\mathrm{o}}=10^{\circ}$ 、后角 $\alpha_{\mathrm{o}}=5^{\circ}$ 、副后角 $\alpha_{\mathrm{o}}^{\prime}=6^{\circ}$ 。

冷风源: CTL-50/3 冷风机, 设定温度 $-30{ }^{\circ} \mathrm{C}$ 、 压力 $0.4 \sim 0.6 \mathrm{MPa}$ 、流量 $3.0 \mathrm{~m}^{3} / \mathrm{min}$, 冷风微油雾( 加油量 $50 \mathrm{~mL} / \mathrm{h}$ )从前刀面射入。

力传感器: Kistler 三分量测力计 $9257 \mathrm{~B}$, 切削 力测量范围 $-5 \mathrm{kN} \sim 5 \mathrm{kN}$, 检测灵敏度 $2.3 \mathrm{pc} / \mathrm{N}$ 。

采集系统: 与力传感器配套的 Dewetron 数据采 集系统 DEWE-3021, 有 16 通道, 每通道最高采样 率 $100 \mathrm{kHZ}$ 。

粗粘度检测设备: SURFCOM480A。温度检测: 固定式 VH-480HS 红外热像仪。应力检测: X-350A 型 $\mathrm{X}$ 射线应力测定仪。数据分析处理软件: 均匀设 计软件 5.0 版。

为了车削试验数据的准确采集记录, 试件设计 成如图 1 所示。在车削试验时, 每个因素水平组合 试验一般会有 多次走刀, 当车刀后刀面磨损值 $V B \geqslant 0.3 \mathrm{~mm}$ 时即停止该次试验。每次试验的每次走 刀到距试件台阶 $5 \mathrm{~mm}$ 位置时检测切削力 $F_{x} 、 F_{y}$ 、 $F_{z}$, 走刀结束时采集切削温度 $T$, 取下试件同样在 距台阶 $5 \mathrm{~mm}$ 位置检测表面粗粘度 $R_{\mathrm{a}}$ 和表面残余应
力 $\sigma_{\mathrm{r}}$, 取下刀片检测后刀面磨损量 $V B$, 每次试验 结束按式(1)计算刀具寿命。

$$
\mathrm{SCL}=\sum_{i=1}^{n} \frac{D_{\mathrm{mi}} \times \pi}{1000} \times \frac{l_{\mathrm{m}}}{f}
$$

式中, SCL 为刀具寿命, 即螺旋切削长度, 单位 $\mathrm{km}$; $D_{\mathrm{m} i}$ 为第 $i$ 次走刀时已加工表面直径, 单位 $\mathrm{mm} ; l_{\mathrm{m}}$ 为试件切削加工长度, 单位 $\mathrm{mm}$, 本试验 $l_{\mathrm{m}}=245.0 \mathrm{~mm} ; n$ 为每次试验走刀次数; $f$ 为进给 量, 单位 $\mathrm{mm} / \mathrm{r}$ 。

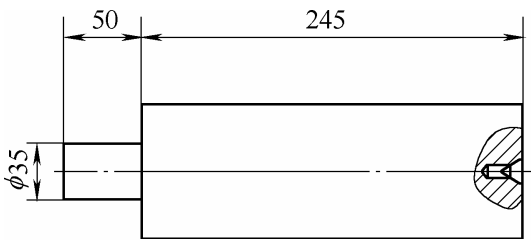

图 1 试件图

用均匀设计软件优化运算产生如表 1 所示奥氏 体不锈钢精密车削试验含定性因素均匀混合水平 表, 该试验设计表格的均匀性中心化偏差 $C_{\mathrm{D} 2}=$ 0.02576 , 非常理想。精密车削试验选定的定量因素 有切削速度 $v$ 、背吃刀量 $a_{\mathrm{p}}$ 、进给量 $f$ 和刀尖半径 $r_{\varepsilon}$; 定性因素为车削冷却降温条件 $A, A$ 是伪变量, 干式车削 $A_{1}$ 用 $\left(A_{1}=1, A_{2}=0\right)$ 表达, 低温冷风微油雾 车削 $A_{2}$ 用 $\left(A_{1}=0, A_{2}=1\right)$ 表达、环保型湿式车削 $A_{3}$ 用 $\left(A_{1}=A_{2}=0\right)$ 表达。精密车削试验方案与试验结果 的刀具磨损情况如表 1 。为了减小检测误差对车削 试验研究结果产生不良影响, 从每次车削试验的刀 具稳定磨损阶段选取 14 组数据(若稳定磨损阶段走 刀次数不够 14 次的则重复一轮试验)作为车削试验 研究的重复试验分析数据, 如表 2 所示(仅列出一组 数据, 切削力仅列 $F_{z}$ )。

刀具寿命 SCL 通过式(1)根据表 1 记录的走刀次 数、每次走刀车削的直径和进给量计算得出, 用 SCL 作为刀具寿命的计量指标可较为客观地反映刀具的 耐磨性，但在金属切削加工中刀具成本往往较低， 一般不会超过 $2 \% \sim 4 \%$, 因此在作精密车削参数优 化时, 本文追求的主要目标不仅仅是刀具寿命 SCL 的长度, 而是在保证工件表面完整性前提下的切削 效率。往往是在精密车削参数优化前就需要明确刀 具能车削出多少个工件的完整表面, 以保证在走刀 车削过程中不换刀，在工件连续表面不出现接刀痕 迹, 在此前提下来提高切削效率。因此有必要提出 在刀具磨钝前能车削出的总金属表面积这个指标作 为刀具寿命, 记为切削表面积 $S, S=\mathrm{SCL} \times f$ 。两种 刀具寿命数据同样列入表 2 。 
表 1 奥氏体不锈钢精密车削试验方案及刀具磨损情况

\begin{tabular}{|c|c|c|c|c|c|c|c|c|}
\hline 编号 & $\begin{array}{c}\text { 切削速度 } \\
v /(\mathrm{m} / \mathrm{min})\end{array}$ & $\begin{array}{c}\text { 背吃刀量 } \\
a_{\mathrm{p}} / \mathrm{mm}\end{array}$ & $\begin{array}{l}\text { 进给速度 } \\
f /(\mathrm{mm} / \mathrm{r})\end{array}$ & $\begin{array}{c}\text { 刀尖半径 } \\
r_{\varepsilon} / \mathrm{mm}\end{array}$ & 冷却降温条件 & 走刀次数 & $\begin{array}{l}\text { 后刀面磨损 } \\
\text { 值 } V B / \mathrm{mm}\end{array}$ & 备注 \\
\hline 1 & 190 & 2.0 & 0.13 & 0.8 & $A_{1}$ & 57 & 0.304 & \\
\hline 2 & 170 & 1.2 & 0.25 & 0.4 & $A_{1}$ & 53 & 0.306 & \\
\hline 3 & 200 & 1.0 & 0.21 & 1.2 & $A_{3}$ & 200 & 0.168 & \\
\hline 4 & 250 & 2.0 & 0.25 & 0.8 & $A_{3}$ & 11 & 刀尖崩裂 & 重复一轮 \\
\hline 5 & 180 & 1.6 & 0.29 & 1.2 & $A_{2}$ & 76 & 刀尖崩裂 & \\
\hline 6 & 210 & 1.8 & 0.33 & 0.4 & $A_{2}$ & 8 & 刀尖崩裂 & 重复一轮 \\
\hline 7 & 270 & 1.8 & 0.21 & 1.2 & $A_{1}$ & 18 & 刀尖崩裂 & \\
\hline 8 & 240 & 1.4 & 0.13 & 1.2 & $A_{2}$ & 74 & 0.314 & \\
\hline 9 & 220 & 1.6 & 0.17 & 0.4 & $A_{3}$ & 20 & 0.326 & \\
\hline 10 & 260 & 1.2 & 0.17 & 0.4 & $A_{2}$ & 14 & 刀尖崩裂 & 重复一轮 \\
\hline 11 & 230 & 1.0 & 0.33 & 0.8 & $A_{1}$ & 13 & 刀尖崩裂 & 重复一轮 \\
\hline 12 & 280 & 1.4 & 0.29 & 0.8 & $A_{3}$ & 10 & 刀尖崩裂 & 重复一轮 \\
\hline
\end{tabular}

表 2 奥氏体不锈钢车削试验数据

\begin{tabular}{|c|c|c|c|c|c|c|c|c|c|c|c|}
\hline $\begin{array}{l}\text { 切削速度 } \\
v /(\mathrm{m} / \mathrm{min})\end{array}$ & $\begin{array}{c}\text { 背吃刀量 } \\
a_{\mathrm{p}} / \mathrm{mm}\end{array}$ & $\begin{array}{l}\text { 进给速度 } \\
f /(\mathrm{mm} / \mathrm{r})\end{array}$ & $\begin{array}{c}\text { 刀尖半径 } \\
r_{\varepsilon} / \mathrm{mm}\end{array}$ & & $\begin{array}{l}\text { 降 } \\
\text { 件 } \\
A_{2} \\
\end{array}$ & $\begin{array}{c}\text { 主切削力 } \\
F_{z} / \mathrm{N}\end{array}$ & $\begin{array}{c}\text { 表面粗 } \\
\text { 糙度 } \\
R_{\mathrm{a}} / \mu \mathrm{m}\end{array}$ & $\begin{array}{l}\text { 表面残 } \\
\text { 余应力 } \\
\sigma_{\mathrm{r}} / \mathrm{MPa}\end{array}$ & $\begin{array}{c}\text { 切削温度 } \\
T /{ }^{\circ} \mathrm{C}\end{array}$ & $\begin{array}{l}\text { 刀具寿命 } \\
\mathrm{SCL} / \mathrm{km}\end{array}$ & $\begin{array}{l}\text { 切削表面 } \\
\text { 积 } S / \mathrm{m}^{2}\end{array}$ \\
\hline 190 & 2.0 & 0.13 & 0.8 & 1 & 0 & 368.7 & 0.823 & 261.7 & 766.29 & 20.627 & 2.682 \\
\hline 170 & 1.2 & 0.25 & 0.4 & 1 & 0 & 383.4 & 2.374 & 105.2 & 710.92 & 9.863 & 2.466 \\
\hline 200 & 1.0 & 0.21 & 1.2 & 0 & 0 & 252.4 & 1.172 & 566.9 & 491.50 & 48.343 & 10.152 \\
\hline 250 & 2.0 & 0.25 & 0.8 & 0 & 0 & 568.0 & 2.166 & 468.7 & 632.50 & 2.321 & 0.580 \\
\hline 180 & 1.6 & 0.29 & 1.2 & 0 & 1 & 518.8 & 2.094 & 684.1 & 504.24 & 11.964 & 3.470 \\
\hline 210 & 1.8 & 0.33 & 0.4 & 0 & 1 & 709.8 & 4.117 & 198.9 & 678.84 & 1.361 & 0.449 \\
\hline 270 & 1.8 & 0.21 & 1.2 & 1 & 0 & 450.4 & 0.978 & 655.2 & 677.56 & 3.788 & 0.795 \\
\hline 240 & 1.4 & 0.13 & 1.2 & 0 & 1 & 253.6 & 0.839 & 526.4 & 530.89 & 24.250 & 3.153 \\
\hline 220 & 1.6 & 0.17 & 0.4 & 0 & 0 & 342.7 & 1.896 & -125.0 & 382.25 & 5.150 & 0.876 \\
\hline 260 & 1.2 & 0.17 & 0.4 & 0 & 1 & 270.6 & 1.482 & -45.7 & 509.06 & 4.138 & 0.703 \\
\hline 230 & 1.0 & 0.33 & 0.8 & 1 & 0 & 328.7 & 3.175 & 761.4 & 722.99 & 1.951 & 0.644 \\
\hline 280 & 1.4 & 0.29 & 0.8 & 0 & 0 & 460.7 & 2.232 & 550.8 & 625.83 & 1.741 & 0.505 \\
\hline
\end{tabular}

\section{2 建立回归模型}

\section{1 多元二次回归模型}

为了很好地反映在三种清洁车削冷却降温条 件下, 切削速度 $v$ 、进给量 $f$ 、背吃刀量 $a_{\mathrm{P}}$ 和刀尖 半径 $r_{\varepsilon}$ 四个自变量及交互作用对主切削力 $F_{z}$ 、表面 粗粘度 $R_{\mathrm{a}}$ 、表面残余应力 $\sigma_{\mathrm{r}}$ 、切削温度 $T$ 和刀具 寿命 $S$ 的影响, 用均匀设计软件 5.0 版按最优回归 子集法进行运算处理, 可得

$$
\begin{aligned}
y_{1}= & 408.174+254.715\left(x_{2}-1.5\right)+ \\
& 1359.497\left(x_{3}-0.229\right)+9.175 A_{1}- \\
& 1.016\left(x_{1}-220\right)\left(x_{2}-1.5\right)+ \\
& 1.217\left(x_{1}-220\right)\left(x_{4}-0.8\right)-1.129\left(x_{1}-220\right) A_{1}+ \\
& 1828.061\left(x_{2}-1.5\right)\left(x_{3}-0.229\right)+ \\
& 192.125\left(x_{3}-0.229\right) A_{2}+100.412\left(x_{4}-0.8\right) A_{1}- \\
& 13.188\left(x_{4}-0.8\right) A_{2}
\end{aligned}
$$

$$
\begin{aligned}
y_{2}= & 2.237+0.226\left(x_{2}-1.5\right)+12.044\left(x_{3}-0.229\right)- \\
& 1.06\left(x_{4}-0.8\right)+0.013 A_{2}-0.0002\left(x_{1}-220\right)^{2}+ \\
& 0.002\left(x_{1}-220\right) A_{2}-1.019\left(x_{2}-1.5\right)^{2}+ \\
& 10.908\left(x_{3}-0.229\right)^{2}- \\
& 4.439\left(x_{3}-0.229\right)\left(x_{4}-0.8\right)- \\
& 0.247\left(x_{4}-0.8\right) A_{1}
\end{aligned}
$$$$
y_{3}=389.362+2360.406\left(x_{3}-0.229\right)+
$$$$
736.609\left(x_{4}-0.8\right)+76.563 A_{1}+
$$$$
0.353\left(x_{1}-220\right)\left(x_{2}-1.5\right)+0.553\left(x_{1}-220\right) A_{1}+
$$$$
66.982\left(x_{2}-1.5\right)^{2}+
$$$$
470.72\left(x_{2}-1.5\right)\left(x_{3}-0.229\right)+
$$$$
5909.492\left(x_{3}-0.229\right)^{2}-
$$$$
1135.879\left(x_{3}-0.229\right) A_{2}-618.341\left(x_{4}-0.8\right)^{2}
$$$$
y_{4}=556.236-0.4\left(x_{1}-220\right)+6.675\left(x_{2}-1.5\right)+
$$$$
340.601 A_{1}+0.024\left(x_{1}-220\right)^{2}-
$$ 


$$
\begin{aligned}
& 0.901\left(x_{1}-220\right)\left(x_{4}-0.8\right)-0.048\left(x_{1}-220\right) A_{2}+ \\
& 5539.881\left(x_{2}-1.5\right)\left(x_{3}-0.229\right)- \\
& 230.072\left(x_{2}-1.5\right)\left(x_{4}-0.8\right)+ \\
& 10863.581\left(x_{3}-0.229\right)^{2}-1180.658\left(x_{4}-0.8\right)^{2} \\
& y_{5}=1.457-0.05\left(x_{1}-220\right)+4.646\left(x_{4}-0.8\right)+ \\
& 0.578 A_{1}+0.001\left(x_{1}-220\right)^{2}- \\
& 24.134\left(x_{2}-1.5\right)\left(x_{4}-0.8\right)- \\
& \text { 0.594( } \left.x_{2}-1.5\right) A_{1}-11.193\left(x_{2}-1.5\right) A_{2}- \\
& 120.544\left(x_{3}-0.229\right)^{2}+4.875\left(x_{4}-0.8\right)^{2}- \\
& 2.931\left(x_{4}-0.8\right) A_{2} \\
& \text { 令 } v=x_{1} 、 a_{\mathrm{p}}=x_{2} 、 f=x_{3} 、 r_{\varepsilon}=x_{4} 、 F_{z}=y_{1} \text { 、 } \\
& \text { 量模型, 如式(2)所述, 且满足以下条件 } \\
& 170 \leqslant v \leqslant 280 \quad 1 \leqslant a_{\mathrm{p}} \leqslant 2 \\
& 0.13 \leqslant f \leqslant 0.33 \quad 0.4 \leqslant r_{\varepsilon} \leqslant 1.2 \\
& A_{1}=0 \quad A_{2}=0 \quad A_{3} \\
& A_{1}=0 \quad A_{2}=1 \quad A_{2} \\
& A_{1}=1 \quad A_{2}=0 \quad A_{1} \\
& F_{\mathrm{z}}=408.174+254.715\left(a_{\mathrm{p}}-1.5\right)+1359.497(f-0.229)+9.175 A_{1}-1.016(v-220)\left(a_{\mathrm{p}}-1.5\right)+ \\
& 1.217(v-220)\left(a_{\mathrm{p}}-1.5\right)-1.129(v-220) A_{1}+1828.061\left(a_{\mathrm{p}}-1.5\right)(f-0.229)+ \\
& 192.125(f-0.229) A_{2}+100.412\left(r_{\varepsilon}-0.8\right) A_{1}-13.188\left(r_{\varepsilon}-0.8\right) A_{2} \\
& R_{\mathrm{a}}=2.237+0.226\left(a_{\mathrm{p}}-1.5\right)+12.044(f-0.229)-1.06\left(r_{\varepsilon}-0.8\right)+0.013 A_{2}-0.0002(v-220)^{2}+ \\
& 0.002(v-220) A_{2}-1.019\left(a_{\mathrm{p}}-1.5\right)^{2}+10.908(f-0.229)^{2}-4.439(f-0.229)\left(r_{\varepsilon}-0.8\right)- \\
& 0.247\left(r_{\varepsilon}-0.8\right) A_{1} \\
& \sigma_{\mathrm{r}}=389.362+2360.406(f-0.229)+736.609\left(r_{\varepsilon}-0.8\right)+76.563 A_{1}+0.353(v-220)\left(a_{\mathrm{p}}-1.5\right)+ \\
& 0.553(v-220) A_{1}+66.982\left(a_{\mathrm{p}}-1.5\right)^{2}+470.72\left(a_{\mathrm{p}}-1.5\right)(f-0.229)+ \\
& 5909.492(f-0.229)^{2}-1135.879(f-0.229) A_{2}-618.341\left(r_{\varepsilon}-0.8\right)^{2} \\
& T=556.236-0.4(v-220)+6.675\left(a_{\mathrm{p}}-1.5\right)+340.601 A_{1}+0.024(v-220)^{2}- \\
& 0.901(v-220)\left(r_{\varepsilon}-0.8\right)-0.048(v-220) A_{2}+5539.881\left(a_{\mathrm{p}}-1.5\right)(f-0.229)- \\
& 230.072\left(a_{\mathrm{p}}-1.5\right)\left(r_{\varepsilon}-0.8\right)+10863.581(f-0.229)^{2}-1180.658\left(r_{\varepsilon}-0.8\right)^{2} \\
& S=1.457-0.05(v-220)+4.646\left(r_{\varepsilon}-0.8\right)+0.578 A_{1}+0.001(v-220)^{2}- \\
& 24.134\left(a_{\mathrm{p}}-1.5\right)\left(r_{\varepsilon}-0.8\right)-0.594\left(a_{\mathrm{p}}-1.5\right) A_{1}-11.193\left(a_{\mathrm{p}}-1.5\right) A_{2}- \\
& 120.544(f-0.229)^{2}+4.875\left(r_{\varepsilon}-0.8\right)^{2}-2.931\left(r_{\varepsilon}-0.8\right) A_{2}
\end{aligned}
$$

\begin{tabular}{|c|c|c|c|c|}
\hline \multicolumn{2}{|c|}{ 来源 } & 回归 & 残差 & 总和 \\
\hline \multirow{5}{*}{ 平方和 } & $F_{z}$ & 213155.0 & 0.1 & 213155.0 \\
\hline & $R_{\mathrm{a}}$ & 10.8 & 0 & 10.8 \\
\hline & $\sigma_{\mathrm{r}}$ & 965438.4 & 0.2 & 965438.5 \\
\hline & $T$ & 150350.4 & 0 & 150350.4 \\
\hline & $S$ & 83.0 & 0 & 83.0 \\
\hline \multirow{5}{*}{ 均方 } & $F_{z}$ & 21315.5 & 0.1 & - \\
\hline & $R_{\mathrm{a}}$ & 1.1 & 0 & - \\
\hline & $\sigma_{\mathrm{r}}$ & 96543.8 & 0.2 & - \\
\hline & $T$ & 15035.1 & 0 & - \\
\hline & $S$ & 8.3 & 0 & - \\
\hline \multirow{5}{*}{$F$ 值 } & $F_{z}$ & 409368.3 & - & - \\
\hline & $R_{\mathrm{a}}$ & 144807.0 & - & - \\
\hline & $\sigma_{\mathrm{r}}$ & 532811.9 & - & - \\
\hline & $T$ & 545400.7 & - & - \\
\hline & $S$ & 750.6 & - & - \\
\hline
\end{tabular}$$
R_{\mathrm{a}}=y_{2} 、 \sigma_{\mathrm{r}}=y_{3} 、 T=y_{4} 、 S=y_{5} \text {, 则有五个因变 }
$$

\section{2 回归显著检验}

对表面粗糖度 $R_{\mathrm{a}}$ 、表面残余应力 $\sigma_{\mathrm{r}}$ 、切削力 $F_{z}$ 、切削温度 $T$ 和刀具寿命 $S$ 五个因变量的方差分 析结果见表 3 , 可见五个因变量的均方与平方和的 残差都很小。若取显著水平为 $\alpha=0.03$, 查 $\mathrm{F}$ 分 布表则有临界值 $F_{0.03}(10,1)=672.546$ 。表面粗糙度 的 $F=144807 \gg 672.546$, 回归效果非常显著; 表 面残余应力的 $F=532811.9 \gg 672.546$, 回归效果 非常显著; 主切削力的 $F=409368.3 \gg 672.546$, 回归效果非常显著; 切削温度的 $F=545400.7 \gg 672.546$, 回归效果非常显著; 刀 具寿命的 $F=750.6>672.546$, 回归效果显著。

\section{3 多目标优化}

\section{1 优化变量}

试验选用肯纳硬质合金涂层刀片, 刀具商已针
表 3 方差分析结果

对不同被切材料做了大量试验研究，刀片几何角度 
已针对不同被切材料的加工精度要求进行了优化设 计, 除刀尖半径以外的几何角度都已固定, 只有刀 尖半径可选。因此本试验研究将切削速度 $v$ 、背吃 刀量 $a_{\mathrm{p}}$ 、进给量 $f$ 、刀尖半径 $r_{\varepsilon}$ 和冷却降温条件 $A$ 作为优化变量, 五个优化变量可看作五维欧式空间 $E^{5}$ 中的一个点, 相应优化变量可表示为

$$
x=\left(v, a_{\mathrm{p}}, f, r_{\varepsilon}, A\right)=\left(x_{1}, x_{2}, x_{3}, x_{4}, x_{5}\right) \in E^{5}
$$

\section{2 目标函数}

选定的精密车削参数优化目标有: 切削效率 $Q^{\prime}$ 或 $S^{\prime}$ 、刀具寿命 $\mathrm{SCL}$ 或 $S$ 、表面粗粘度 $R_{\mathrm{a}}$ 、表面 残余应力 $\sigma_{\mathrm{r}}$ 、切削力 $F_{z}$ 和切削温度 $T$ 。这六个目标 可表达成如下六个目标函数。

(1) 切削效率。在金属切削加工中, 粗加工的 效率追求目标是尽快切除毛坏余量, 而精密加工的 效率追求目标则是尽快形成符合技术要求的表面 积。因此, 粗加工的切削效率常用单位时间内切除 的金属体积 $Q^{\prime}$ 作为目标函数, 而精密加工的切削效 率则常用单位时间内形成的表面积 $S^{\prime}$ 作为目标函 数。本车削试验研究用表面切出率 $S^{\prime}$ 作目标函数

$$
S^{\prime}=1000 v f=f_{1}(v, f)=f_{1}(x)
$$

切削效率优化的目标取极大值。

(2) 刀具寿命。精密车削的刀具寿命, 可用切 削表面积 $S$ 作为目标函数

$$
\begin{gathered}
S=f \times \mathrm{SCL}=f \sum_{i=1}^{n} \frac{D_{\mathrm{m} i} \times \pi}{1000} \times \frac{l_{\mathrm{m}}}{f}= \\
f_{2}\left(v, a_{\mathrm{p}}, f, r_{\varepsilon}, A\right)=f_{2}(x)
\end{gathered}
$$

对精密车削, 在车削参数优化前就需要明确刀 具能车削出多少个工件完整表面, 以保证在一次车 削走刀过程中不换刀, 因此可将其作为约束函数。

(3) 表面粗粘度

$$
R_{\mathrm{a}}=f_{3}\left(v, a_{\mathrm{p}}, f, r_{\varepsilon}, A\right)=f_{3}(x)
$$

表面粗粘度优化的目标过去一般是取极小值, 但现代制造技术经济学观点, 保证的工艺技术要求 的粗楉度即可, 不必追求极小。因此, 表面粗粘度 也可作为约束函数。

(4) 表面残余应力

$$
\sigma_{\mathrm{r}}=f_{4}\left(v, a_{\mathrm{p}}, f, r_{\varepsilon}, A\right)=f_{4}(x)
$$

表面残余应力为 “正” 时, 属于拉应力, 极其 容易引起表面的微裂纹, 而降低零件的疲劳寿命; 表面残余应力为 “负” 时, 属于压应力, 非常有益 于疲劳寿命的提高。但从现代制造技术经济学观点, 表面残余应力在一定范围即可, 因此表面残余应力 也可作为约束函数。
(5) 切削力。精密车削试验检测有切削力的三 个分力 $F_{x} 、 F_{y} 、 F_{z}$, 为了简化精密车削参数优化 目标，本文仅选主切削力 $F_{z}$ 作为目标函数

$$
F_{z}=f_{5}\left(v, a_{\mathrm{p}}, f, r_{\varepsilon}, A\right)=f_{5}(x)
$$

切削力的大小对工艺系统变形有较大负面影 响, 切削力优化目标取极小值。

(6) 切削温度

$$
T=f_{6}\left(v, a_{\mathrm{p}}, f, r_{\varepsilon}, A\right)=f_{6}(x)
$$

切削温度对工艺系统工作稳定性、切削效率和 表面完整性的影响较为复杂，一般情况下车削参数 优化目标应该是切削温度在一定范围内。基于本试 验是高速车削, 切削温度较高, 切削温度优化目标 取极小值。

上面六个目标函数可抽象为 $\left(f_{1}(x), f_{2}(x), f_{3}(x)\right.$, $\left.f_{4}(x), f_{5}(x), f_{6}(x)\right)^{\mathrm{T}}$

从现代制造技术经济学角度出发考虑将 $f_{2}(x)$ 、 $f_{3}(x) 、 f_{4}(x)$ 作为约束函数, 相应目标函数可抽 象为

$$
\left(f_{1}(x), f_{5}(x), f_{6}(x)\right)^{\mathrm{T}}
$$

\section{3 约束函数}

在精密车削优化试验中, 机床功率、主轴扭矩、 主轴转速、进给速度、刀具强度及几何角度、切削 温度、切削冷却降温条件等因素构成参数优化的约 束函数。在精密车削试验方案设计时, 一部分约束 已通过确定优化变量的取值范围得到体现, 另一部 分约束直接在数学模型中体现。约束函数可表达为

$$
g_{\mathrm{i}}\left(v, a_{\mathrm{p}}, f, r_{\varepsilon}, A\right)=g_{\mathrm{i}}(x) \leqslant 0 \quad i=1,2, \cdots, m
$$

表面粗粮度、表面残余应力、刀具寿命、优化 变量取值范围和车削冷却降温条件分别表达为 $g_{1}(x) 、 g_{2}(x) 、 g_{3}(x) 、 g_{4}(x) 、 g_{5}(x)$ 。

\section{4 优化数学模型}

若记：优化目标函数为

$$
f(x)=\left(f_{1}(x), f_{5}(x), f_{6}(x)\right)^{\mathrm{T}}
$$

优化约束函数为

$$
g(x)=\left(g_{1}(x), g_{2}(x), g_{3}(x), g_{4}(x), g_{5}(x)\right)^{\mathrm{T}}
$$

则约束空间为 $R=\left\{x \in E^{5} \mid g(x) \leqslant 0\right\}$ 。切削效率 $S^{\prime}$ 优化目标取极大值、切削力 $F_{z}$ 和切削温度 $T$ 优化 目标取极小值, 则精密车削参数优化模型可表达为

$$
\begin{gathered}
\min f(x)=\min \left(-f_{1}(x), f_{5}(x), f_{6}(x)\right)^{\mathrm{T}} \\
x \in R
\end{gathered}
$$

工程实际中, 由工艺技术要求给定表面粗糙度 
和表面残余应力取值范围, 即 $a \leqslant R_{\mathrm{a}} \leqslant b$ 、 $c \leqslant \sigma_{\mathrm{r}} \leqslant d$; 刀具寿命 $S$ 根据工程实践经验初步提出 再经试切而最终确定, $S$ ? eph, $e$ 为安全系数, 以 保证刀具在正常磨损阶段工作， $p$ 为刀具磨钝前能 车削出的工件个数, $h$ 为工件的车削表面积。约束 空间可具体化为

$$
R^{\prime}=\left\{x \in R \left\{\begin{array}{l}
a \leqslant R_{\mathrm{a}} \leqslant b \\
c \leqslant \sigma_{\mathrm{r}} \leqslant d \\
S \geqslant e p h \\
170 \leqslant v \leqslant 280 \\
1.0 \leqslant a_{\mathrm{p}} \leqslant 2.0 \\
0.13 \leqslant f \leqslant 0.33 \\
0.4 \leqslant r_{\varepsilon} \leqslant 1.2 \\
A_{1}=0 \quad A_{2}=0 \\
A_{1}=0 \quad A_{2}=1 \\
A_{1}=1 \quad A_{2}=0
\end{array}\right.\right.
$$

精密车削参数优化模型可转化为

$$
\begin{gathered}
\min \left(-S^{\prime}, F_{z}, T\right)^{\mathrm{T}} \\
x \in R^{\prime}
\end{gathered}
$$

为了方便现场运用, 可将模型具体化表达为

$$
\left\{\begin{array}{l}
-k S^{\prime}+l F_{\mathrm{z}}+m T=\min \\
a \leqslant R_{\mathrm{a}} \leqslant b \\
c \leqslant \sigma_{\mathrm{r}} \leqslant d \\
S \geqslant e p h \\
170 \leqslant v \leqslant 280 \\
1.0 \leqslant a_{\mathrm{p}} \leqslant 2.0 \\
0.13 \leqslant f \leqslant 0.33 \\
0.4 \leqslant r_{\varepsilon} \leqslant 1.2 \\
A_{1}=0 \quad A_{2}=0 \quad A_{3} \\
A_{1}=0 \quad A_{2}=1 \quad A_{2} \\
A_{1}=1 \quad A_{2}=0 \quad A_{1}
\end{array}\right.
$$

式中, $k, l, m$ 为权系数, 可根据优化的侧重而确定 具体权值。

\section{4 优化模型验证}

同样以图 1 所示试件作为验证件, 要求车削到 $\phi 60.00 \mathrm{~mm}$, 留有车削余量 $1.0 \mathrm{~mm}$, 表面粗粘度控 制在 $0.8 \mu \mathrm{m} \leqslant R_{\mathrm{a}} \leqslant 1.6 \mu \mathrm{m}$ 内, 表面残余应力控制在 $80 \mathrm{MPa} \leqslant \sigma_{\mathrm{r}} \leqslant 300 \mathrm{MPa}$ 内, 要求至少车削加工 50 件才能换刀。
根据上述技术要求可确定: $a=0.8, b=1.6$, $c=80, d=300, p=50, e=1.2, h=0.046$ 。

通过调试三个权系数 $k 、 l 、 m$ 进行优化运算, 其结果优化变量分别叙述如下。

干 式 车 削 $A_{1}(193.1,1.0,0.21,0.49)$, 即 $v=193.1 \mathrm{~m} / \mathrm{min} 、 a_{\mathrm{P}}=1.0 \mathrm{~mm} 、 f=0.2 \mathrm{~mm} / \mathrm{r}$ 、 $r_{\varepsilon}=0.49 \mathrm{~mm}$ 。

低温冷风微油雾车削 $A_{2}(216.2,1.0,0.23,0.85)$, 即 $v=216.2 \mathrm{~m} / \mathrm{min} 、 a_{\mathrm{P}}=1.0 \mathrm{~mm} 、 f=0.23 \mathrm{~mm} / \mathrm{r}$ 、 $r_{\varepsilon}=0.85 \mathrm{~mm}$ 。

环保型湿式车削 $A_{3}(238.6,1.0,0.19,0.76)$, 即 $v=238.6 \mathrm{~m} / \mathrm{min} 、 a_{\mathrm{P}}=1.0 \mathrm{~mm} 、 f=0.19 \mathrm{~mm} / \mathrm{r}$ 、 $r_{\varepsilon}=0.76 \mathrm{~mm}$ 。

因刀尖半径受尺寸系列 $\left(r_{\varepsilon}=0.4,0.8,1.2\right)$ 限制, 需圆整刀尖半径后使其固定再做预测。

最终结果优化变量分别综合如下。

干式车削 $A_{1}(183,1.0,0.22,0.4)$ 即 $v=183 \mathrm{~m} / \mathrm{min}$ 、 $a_{\mathrm{P}}=1.0 \mathrm{~mm} 、 f=0.22 \mathrm{~mm} / \mathrm{r} 、 r_{\varepsilon}=0.4 \mathrm{~mm}$ 。

低温冷风微油雾车削 $A_{2}(223,1.0,0.21,0.8)$, 即 $v=223 \mathrm{~m} / \mathrm{min} 、 a_{\mathrm{P}}=1.0 \mathrm{~mm} 、 f=0.21 \mathrm{~mm} / \mathrm{r}$ 、 $r_{\varepsilon}=0.8 \mathrm{~mm}$ 。

环保型湿式车削 $A_{3}(253,1.0,0.19,0.8)$ ， 即 $v=253 \mathrm{~m} / \mathrm{min} 、 a_{\mathrm{P}}=1.0 \mathrm{~mm} 、 f=0.19 \mathrm{~mm} / \mathrm{r}$ 、 $r_{\varepsilon}=0.8 \mathrm{~mm}$ 。

在上述优化变量下, 六个因变量的预测结果及 验证见表 4 , 可见干式车削的表面切出率 $S^{\prime}=0.0677 \mathrm{~m}^{2} / \mathrm{min}, 40.8 \mathrm{~s}$ 可车削完一个工件, 在 刀具磨针前可车削出 62 个工件, 建议车削出 62 个 工件换刀; 低温冷风微油雾车削的表面切出率 $S^{\prime}=0.0741 \mathrm{~m}^{2} / \mathrm{min}, 37.3 \mathrm{~s}$ 可车削完一个工件, 在 刀具磨针前可车削 61 个工件, 建议车削出 61 个工 件换刀; 环保型湿式车削的表面切出率 $S^{\prime}=0.0805 \mathrm{~m}^{2} / \mathrm{min}, 34.3 \mathrm{~s}$ 可车削完一个工件, 在 刀具磨针前可车削 207 个工件, 建议车削出 207 个 工件换刀。从表 4 还可发现预测值与验证值的误差 在 $15 \%$ 以内, 且表面粗糙度和表面残余应力的验证 值在工艺技术要求范围内。

\section{5 结论}

（1）均匀设计法设计的含定性因素混合水平的 精密车削试验, 用最优回归子集法建立的各因变量 二次回归模型，其回归效果非常显著。

（2）在各因变量二次回归模型基础上建立的精 密车削参数优化模型, 预测误差在 $15 \%$ 以内。 
表 4 参数优化及验证

\begin{tabular}{|c|c|c|c|c|c|c|c|c|c|c|c|}
\hline \multirow{2}{*}{$\begin{array}{l}\text { 冷却降 } \\
\text { 温条件 }\end{array}$} & \multirow{2}{*}{$\begin{array}{l}\text { 表面切出率 } \\
S^{\prime} /\left(\mathrm{m}^{2} / \mathrm{min}\right)\end{array}$} & \multicolumn{2}{|c|}{ 主切削力 $F_{\mathrm{z}} / \mathrm{N}$} & \multicolumn{2}{|c|}{ 粗楉度 $R_{\mathrm{a}} / \mu \mathrm{m}$} & \multicolumn{2}{|c|}{ 湿度 $T /{ }^{\circ} \mathrm{C}$} & \multicolumn{2}{|c|}{ 应力 $\sigma_{\mathrm{r}} / \mathrm{MPa}$} & \multicolumn{2}{|c|}{ 面积 $S / \mathrm{m}^{2}$} \\
\hline & & 预测值 & 验证值 & 预测值 & 验证值 & 预测值 & 验证值 & 预测值 & 验证值 & 预测值 & 验证值 \\
\hline$A_{1}$ & 0.0677 & 319.9 & 289.8 & 1.297 & 1.175 & 733.6 & 684.6 & 170.9 & 187.6 & 2.86 & 未验证 \\
\hline$A_{2}$ & 0.0741 & 316.6 & 320.4 & 1.351 & 1.182 & 420.4 & 484.5 & 190.1 & 204.3 & 2.85 & 未验证 \\
\hline$A_{3}$ & 0.0805 & 311.7 & 275.7 & 1.198 & 1.233 & 406.8 & 426.6 & 285.5 & 295.6 & 9.56 & 未验证 \\
\hline
\end{tabular}

(3) 在精密车削试验参数范围内, 环保型湿式 车削的刀具寿命很长, 而低温冷风微油雾车削和干 式车削的刀具寿命相对较短。

(4) 在精密车削试验参数范围内, 环保型湿式 车削的切削效率最高, 干式车削的切削效率最低。

(5) 在精密车削试验参数范围内, 低温冷风微 油雾车削和干式车削的刀具寿命都较短, 低温冷风 微油雾车削的刀具寿命影响因素值得进一步研究, 尤其是油雾微粒大小与加油量值得深入研究。

\section{参 考 文 献}

[1] 李登万. 两种难加工材料的切削试验研究及其工艺参 数优化[D]. 成都: 西南交通大学, 2012 .

LI Dengwan. Researches on cutting tests and cutting parameter optimations of two hard-to-cut materials[D]. Chengdu: Southwest Jiaotong University, 2012.

[2] 李波. 空间润滑谐波减速器传动性能正交试验分析 [J]. 机械工程学报, 2012, 48(3): 82-87.

LI Bo. Orthogonal experiment analysis on transmission performance of space lubricated harmonic drive[J]. Journal of Mechanical Engineering, 2012, 48(3): 82-87.

[3] 李先广, 刘飞, 曹华军. 齿轮加工机床的绿色设计与制 造技术[J]. 机械工程学报, 2009, 45(11): 140-145.

LI Xianguang, LIU Fei, CAO Huajun. Green design and manufacturing technology of gear cutting machine [J]. Journal of Mechanical Engineering, 2009, 45(11): 140-145.

[4] 李登万, 陈洪涛, 甘建水, 等. 钛合金加工切削力试验 研究[J]. 广西大学学报, 2010, 35(5): 733-737.

LI Dengwan, CHEN Hongtao, GAN Jianshui, et al.Test study on cutting force in titanium alloy machining[J]. Journal of Guangxi University, 2010，35(5): 733-737.

[5] 横川和彦, 横川宗彦. 环境にゃさしい冷风加工技术[J]. 机械と工具, 1998(7): 46-56.

KAZUHIO Y, MUNEHIK Y. Environment-friendly cold blast machining technology[J]. Tools and Machinery, 1998(7): 46-56.

[6] 李响, 李为吉, 彭程远. 基于均匀试验设计的响应面方 法及其在无人机一体化设计中的应用 [J]. 机械科学与 技术，2005，24(5): 575-577，619.

LI Xiang, LI Weiji, PENG Chengyuan. Response surface methodology based on uniform design and its application to complex engineering system optimization[J]. Mechanical Science and Technology, 2005，24(5): 575-577, 619.

[7] 李晓燕, 张曙, 余灯广. 三维打印成形粉末配方的优化 设计 [J]. 机械科学与技术, 2006, 25(11): 1343-1346.

LI Xiaoyan, ZHANG Shu, YU Dengguang. Optimal design of powder mixtures for three dimensional printing[J]. Mechanical Science and Technology, 2006, 25(11): 1343-1346.

[8] 李登万, 陈洪涛, 许明恒, 等. 基于均匀设计法的钛合 金切削参数优化试验研究 [J]. 机械科学与技术, 2011, 30(5): 689-692.

LI Dengwan, CHEN Hongtao, XU Mingheng, et al. A test study of the cutting parameters optimization of titanium alloy based on uniform design method[J]. Mechanical Science and Technology, 2011, 30(5): 689-692.

[9] CHEHARON C, GINTNG A, ARSHAD H. Pergormance of titanium alloy Ti-6242s[J]. Journal of Materials Processing Technology, 2007, 185: 77-82.

[10] HARTUNG P, KRAMER B. Massachusetts institute of technology[J]. Tool Wear in Titanium Machining, Annals of the CIRP, 1982，31: 75-80.

[11] 李忠群, 刘强. 圆角铣削颤振稳定域建模与仿真研究 [J]. 机械工程学报, 2010, 46(7): 181-186.

LI Zhongqun, LIU Qiang. Modeling and simulation of chatter stability for circular millng[J]. Journal of Mechanical Engineering, 2010，46(7): 181-186.

[12] 张磊, 张志胜, 周一帆, 等. 基于半参数回归模型的制 造过程加工误差流建模与分析 [J]. 机械工程学报, 2013, 49(15): 181-185.

ZHANG Lei, ZHANG Zhisheng, ZHOU Yifan, et al. Stream of variation modeling and analysis for manufacturing processes based on a semi-parametric regression model[J]. Journal of Mechanical Engineering, 2013, 49(15): 181-185.

作者简介: 李登万(通信作者), 男, 1964 年出生, 博士, 教授级高工。 主要研究方向为绿色制造。

E-mail:1dw@scetc.net

陈洪涛, 男, 1967 年出生, 博士, 教授。主要研究方向为数控控制加工 技术。

E-mail: cht@scetc.net

冯锦春, 女, 1971 年出生, 硕士, 教授。主要研究方向为设备控制技术。 E-mail: catsmiling@163.com 